Sharpe, M. E. (1955). J. gen. Microbiol. 12, 107-122.

\title{
A Serological Classification of Lactobacilli
}

\author{
By M. ELISABETH SHARPE \\ National Institute for Research in Dairying, University of Reading
}

SUMMARY : A collection of 442 strains of lactobacilli, including representatives of all recognized species and $\mathbf{1 9 0}$ freshly isolated strains was studied serologically. Using sera prepared against some of these strains and crude $\mathrm{HCl}$ extracts, it was possible to classify $312(70 \%)$ of these strains by precipitin tests into six groups and one subgroup. This classification was in agreement with one based on the physiological characteristics of the strains.

When the serological identification of lactobacilli has been studied in the past, workers have concentrated mainly on limited aspects of the problem rather than on a broad study of the genus, and no comprehensive serological classification has yet been achieved. Results of earlier work in the different fields show conflicting or inconclusive results and the antigenic relations among lactobacilli cannot be deduced from them. More recently Williams $(1948 a, b)$, using agglutinin-absorption tests, investigated antigenic components in oral lactobacilli, and found four major antigens which he designated alphabetically $(\mathrm{A}, \mathrm{B}, \mathrm{C}, \mathrm{D})$, and by monospecific absorbed sera was able to give antigenic analyses for 18 of his 30 strains. Orland (1950) extended the antigenic analyses and was able to detect major antigens in 33 of the 252 strains studied; 30 of these, many of them designated as different species, possessed a common antigen F. Other major antigens were also distinguished, making, with those of Williams, a total of nine major antigens which could be identified in lactobacilli. Although minor antigens were observed in some strains, they could not be demonstrated consistently and the rest of Orland's strains remained unidentified.

Agglutination tests, and occasionally complement-fixation tests, have been used for serological studies of lactobacilli. The precipitin test has been used only infrequently, chiefly by Harrison and his co-workers (Harrison, Zidek \& Hemmens, 1939; Harrison \& Opal, 1944), who used this method to classify oral and intestinal strains of lactobacilli. They extracted a carbohydrate from the lactobacilli by acid extraction, purified it by reprecipitation with ethanol and used a solution of the residue as antigen against their precipitating sera. By quantitative precipitin tests they divided their strains into four serological types and a remaining heterogeneous group of unclassified strains. Niven, Castellani \& Allanson (1949) used ring precipitin tests to identify strains of lactobacilli isolated from spoiled sausage meat. Although $\mathrm{HCl}$ extracts of all their 20 strains reacted with sera prepared against 2 of these strains, they concluded that this was a type reaction.

The purpose of the present work was to investigate serologically strains of lactobacilli from a wide variety of sources, comprising named strains of all 
available species and freshly isolated strains of different origin. Preliminary studies of the antigenic reactions of lactobacilli included both agglutination and precipitin tests. Agglutination tests suggested a large number of heterologous organisms, and were considered to indicate type relationships; precipitin tests, however, indicated broader groups, usually compatible with physiological characteristics, and this method was used for further work.

\section{METHODS}

Organisms. The 442 strains of lactobacilli used for this work were described by Briggs (1953a) and included representatives of all available recognized species. They consisted of a collection of named strains maintained at this Institute, named strains obtained from the laboratory collections of other workers, and freshly isolated strains from different sources. Briggs (1953a) found that 390 out of $\mathbf{4 5 2}$ strains could be separated by physiological tests into eight groups, and the remaining sixty-two organisms into four sections. As these physiological groups of Briggs will be constantly referred to in the text, Table 1 summarizes the biochemical characteristics by which she defined her groups. In the sequel, in designating cultures, NCTC refers to the National Collection of Type Cultures, Colindale, London; NCIB to the National Collection of Industrial Bacteria, Teddington; ATCC to the American Type Culture Collection, Washington, D.C. and NIRD to the collection at the National Institute for Research in Dairying.

Table 1. Physiological reactions characterizing the groups and sections defined by Briggs (1935a)

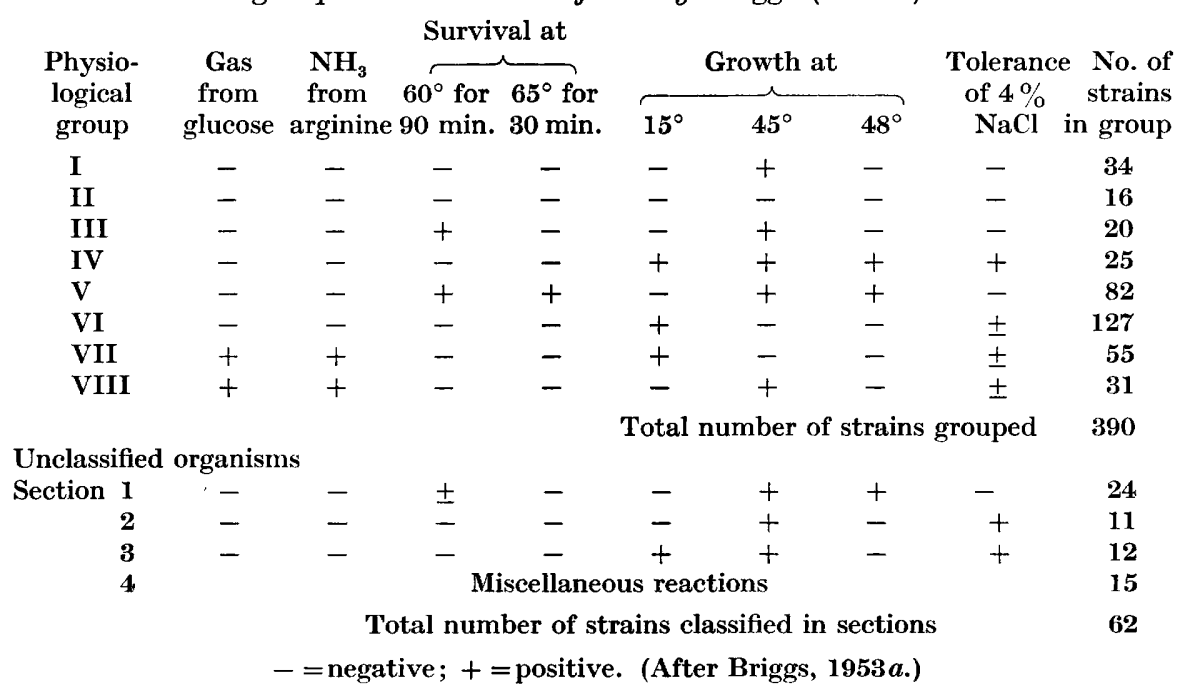

Culture media. At first cultures to be used for immunization and for preparing $\mathrm{HCl}$ extracts were grown in a tomato Yeastrel broth containing Tween 80 (Briggs, 1953b). However, rabbits inoculated with thoroughly washed suspensions of lactobacilli grown in this medium produced antibodies against 
tomato juice and Yeastrel, in addition to specific lactobacillus antibodies. These sera reacted with extracts of all lactobacilli, and with uninoculated media. To eliminate these non-specific reactions, which masked the specific lactobacillus precipitin reactions, cultures to be inoculated into rabbits were grown in medium $\mathbf{A}$ (below) which contained Yeastrel but no tomato juice, and cultures to be used to prepare $\mathrm{HCl}$ extracts in medium $\mathrm{B}$ which contained tomato juice but no Yeastrel. Antibodies to Yeastrel could thus be disregarded as no Yeastrel antigen was present in the extracts. Both media gave an adequate growth of all strains of lactobacilli.

Medium $A(\%, \mathrm{w} / \mathrm{v})$ : Yeastrel, $0 \cdot 3$; glucose, $2 \cdot 0$; NaCl, 0.5; Tween 80, 0.01; Evans peptone, 1.5; final pH value 6·4-6.6. Evans peptone (Evans Medical Supplies Ltd., Liverpool 19) was found to be the most satisfactory for serological work with lactobacilli.

Medium B. This was the same as medium A except that $10.0 \%(\mathrm{v} / \mathrm{v})$ tomato juice replaced the Yeastrel.

Incubation temperatures. Strains of named lactobacilli were incubated at the following temperatures: Lactobacillus acidophilus, L. bulgaricus, L. delbrueckii, L. fermenti, L. helveticus, L. lactis, L. leichmannii, L. odontolyticus at $37^{\circ}$; L. casei, L. plantarum, L. brevis and L. pastorianus at $30^{\circ}$. Freshly isolated strains were incubated at temperatures appropriate to their sources until identified.

\section{Preparation of group sera}

Cultures of lactobacilli grown for $18 \mathrm{hr}$. in $40 \mathrm{ml}$. of medium A were centrifuged, washed twice in $0.85 \%$ saline and suspended in 5-10 ml. saline. Suspensions were heated at $60^{\circ}$ for $1 \mathrm{hr}$., stored at $4^{\circ}$ and used for one course of injections. Suspensions were diluted to an equivalent of Brown's opacity tube no. 3 (Burroughs Wellcome and Co. Ltd.) and rabbits were given a course of five intravenous inoculations at three- to four-day intervals. If a test bleeding then indicated a satisfactory group serum, the rabbit was bled. If not, a fresh suspension of cells was prepared and one or more further courses of inoculation given. Sometimes group antibodies appeared after one course of inoculations, at others two or three courses were necessary, varying with the group and the strain used.

Group sera were tested for potency and specificity, and then stored at $4^{\circ}$ with thiomersalate $(1$ in 10,000$)$ as a preservative; potency was lost on storage, particularly with weak sera. Recently all freshly prepared sera have been freeze-dried within a few weeks of collection, and the results indicate that on reconstitution no loss of potency of group antibodies has occurred. In the earlier part of this work test bleedings were done on all rabbits before inoculation, and the sera tested against extracts of several different strains of lactobacilli for the presence of naturally occurring antibodies. As the results were always negative this practice was discontinued.

As group sera were not always easy to prepare some of the difficulties encountered are mentioned here. Inoculation of some strains of lactobacilli into rabbits gave antisera which reacted only with the homologous extract and sometimes with a few other extracts. Such sera, which were readily produced 
after only a few inoculations, and which gave massive precipitin rings, were therefore regarded as type sera. After further courses of inoculation with some strains group antibodies appeared but with other strains none was detected even after several courses of inoculation; eventually non-specific antibodies common to many unrelated strains of lactobacilli were produced. Some strains, such as those from the NCTC or ATCC, which had been cultivated in vitro for some years and appeared to have lost most or all of their type antigens, gave better group sera than freshly isolated strains which usually gave potent type sera. Strains which produced only type sera reacted, however, with their specific group sera, indicating that group antigen was present.

In some instances the incubation temperature of cultures for inoculation was important, one temperature favouring the formation of group antigen and another the formation of type antigen. Lactobacillus helveticus cultures grown at $37^{\circ}$ stimulated the production of a predominantly type serum; with organisms grown at $30^{\circ}$ a potent group serum with less type activity resulted. With L. casei, on the other hand, incubation at $37^{\circ}$ gave cells which favoured the formation of group antibodies in the serum, whereas incubation at $30^{\circ}$ gave cells which induced the appearance of type antibodies.

Sometimes the number of inoculations necessary to produce group sera yielded sera which also cross-reacted with extracts of strains from other groups. The fact that such antibodies took longer to appear in the sera, and gave weak and irregular reactions with extracts of different strains in a group, suggested that they were due to minor non-specific antigens, which Williams (1948a) and Orland (1950) noted. A knowledge of the behaviour of individual strains and careful control of antibody production by frequent test bleedings usually overcame this trouble, although such cross-reactions could be removed from sera by absorption with suitable strains. Unabsorbed grouping sera were used whenever possible, because it was found on several occasions that absorption with suspensions of heterologous strains greatly weakened the group antibody of a serum even though it gave no precipitin reaction with extracts of the absorbing strain.

It was sometimes found, when test bleedings had indicated that only a weak group antibody had been produced and another course of inoculations was necessary, that after another course of inoculations the group antibodies disappeared leaving only type antibodies, and further injections would not bring back the group reaction. A similar observation was made by Jacob (1947) whilst preparing sera against group E streptococci.

Absorption of sera. Cells for absorbing sera were grown for $48 \mathrm{hr}$. in medium B (chosen because it gave a more profuse growth than medium A), washed twice in saline and the packed cells added to serum in the ratio of 1 vol. cells : 4 vol. serum. Sera were absorbed for $2 \mathrm{hr}$. at $37^{\circ}$ followed by overnight refrigeration; if necessary they were reabsorbed with fresh cells.

\section{Preparation of extracts}

Several different methods of extracting the group antigens from the cells were tried: formamide (Fuller, 1938); trichloroacetic acid (van den Ende, 1952); disintegration of the cells on a tissue disintegrator (Mickle, 1948; cf. 
Shattock, 1949); Lancefield's (1933) $\mathrm{HCl}$ extraction method. The last was the most satisfactory and was used throughout this work. Cells for extraction were grown for $48 \mathrm{hr}$. in $40 \mathrm{ml}$. medium B, centrifuged and extracted with 1.0-2.0 ml. 0.05 $\mathrm{N}-\mathrm{HCl}$ in saline in a boiling water-bath. Extracts were neutralized, thiomersalate (to 1 in 10,000) added and stored at $4^{\circ}$ in stoppered tubes.

Ethanol precipitation of crude $\mathrm{HCl}$ extracts. As described by Harrison et al. (1939) a few of the crude $\mathrm{HCl}$ extracts were purified by precipitating with 4 vol. ethanol, the precipitate was then dissolved in saline, and any undissolved precipitate removed by centrifuging (fraction $A$ ); the ethanol supernatant was evaporated to dryness and redissolved in saline (fraction $\mathrm{B}$ ).

\section{Precipitin tests}

Extracts were layered on serum in small glass tubes of $3.0 \mathrm{~mm}$. internal diameter and kept at room temperature. The junction was examined for ring formation at intervals up to $30 \mathrm{~min}$.

\section{RESULTS}

On testing crude $\mathrm{HCl}$ extracts of lactobacilli against specific grouping sera by the precipitin test it was found possible to classify 312 of the 442 strains examined into six groups and one subgroup. The following serological groups, named after the species from which the grouping sera were prepared were found: bulgaricus, casei-helveticus, casei, fermenti, plantarum and lactisbrevis, subgroup brevis. Table 2 shows the number of strains in each group.

Table 2. Serological grouping of strains of lactobacilli by precipitin tests, using specific group sera

Precipitin reactions of $\mathrm{HCl}$ extracts against specific sera of each serological group of lactobacilli

\begin{tabular}{|c|c|c|c|c|c|c|c|}
\hline \multirow{2}{*}{$\begin{array}{l}\text { Number } \\
\text { of strains } \\
\text { reacting } \\
\text { with sera }\end{array}$} & \multirow[b]{2}{*}{ bulgaricus } & \multirow[b]{2}{*}{$\begin{array}{c}\text { casei- } \\
\text { helveticus }\end{array}$} & \multirow[b]{2}{*}{ casei } & \multirow[b]{2}{*}{ fermenti } & \multirow[b]{2}{*}{ plantarum } & \multicolumn{2}{|c|}{ lactis-brevis } \\
\hline & & & & & & $\begin{array}{l}\text { Unabsorbed } \\
\text { serum }\end{array}$ & $\begin{array}{c}\text { Absorbed } \\
\text { serum }\end{array}$ \\
\hline 26 & + & - & - & - & - & - & - \\
\hline 45 & - & + & - & - & - & - & - \\
\hline 14 & - & - & + & - & - & - & - \\
\hline 22 & - & - & - & + & - & - & - \\
\hline 79 & - & - & - & - & + & - & - \\
\hline 83 & - & - & - & - & - & + & + \\
\hline 43 & - & - & - & - & - & + & - \\
\hline 312 To & & & & & & & \\
\hline
\end{tabular}

\section{LACTOBACILLUS BULGARICUS GROUP}

Group sera were prepared from Lactobacillus bulgaricus NCTC 2889 (ATCC 531) and from $L$. bulgaricus strain Icbna (received from Prof. Sherman). Reciprocal absorption tests showed that these two organisms possessed the same group antigen but a different type antigen. 
Twenty-six strains of lactobacilli fell into this group, $\mathrm{HCl}$ extracts of all of them reacting specifically with Lactobacillus bulgaricus group sera. These 26 organisms consisted of 13 strains named $L$. bulgaricus, 2 of $L$. helveticus, 2 of $L$. acidophilus, and 9 freshly isolated strains from milk, soured milk, présure, and intestine of a pig fed with milk soured with a lactobacillus. According to the physiological tests of Briggs (1953a) 5 of these 26 strains fell into her group I, 8 into group II, 9 into group III, 2 into group V, 1 into group VI and 1 into section 4. Most of them (22 out of 26) are therefore included in groups I, II and III, which, as Briggs pointed out, are not as clearly distinguished from each other as some of her other groups. Since then Wheater (1955), working on the same collection of strains, has differentiated L. acidophilus from $L$. bulgaricus by carbohydrate fermentations, salt tolerance and resistance to bile; 23 of the 26 strains in the present serological group L. bulgaricus were included in Wheater's species $L$. bulgaricus.

\section{LACTOBACILLUS CASEI-HELVETICUS GROUP}

Extracts of 45 strains of lactobacilli gave a specific reaction with a serum prepared with Lactobacillus casei NIRD $\mathrm{R} 094$ as antigen, and also with a serum prepared against $L$. helveticus ATCC 7469 (NCTC 6375). Reciprocal absorption tests (Table 3) showed that these two strains possessed the same group antigen but that each had its own type antigen. Absorption of the group sera with cells of either NIRD R 094 or of ATCC 7469 removed the group but not the type antibodies. As specific sera for this group were prepared from strains named both $L$. casei and $L$. helveticus, the serological group was named L. casei-helveticus. Absorption tests with cells of $L$. casei $\mathrm{H831}$, one of the strains used to prepare serum for the $L$. casei group serum, were also included as controls, and showed that L. casei $\mathbf{H} 831$ had no antigenic relationship with the $L$. casei-helveticus group.

It was found that 28 of the strains falling into the Lactobacillus caseihelveticus group possessed the same type antigen as L. helveticus ATCC 7469. Table 3 shows that when a type-specific serum was prepared (by a very short course of inoculations) with this strain only, strains having antigens of that type reacted; absorption of the serum with cells of NIRD R 094 had no effect, whereas absorption with ATCC 7469 cells removed the type antibodies. The 16 strains which did not possess the ATCC 7469 type antigen were found to have at least 3 other type antigens.

The possession of the ATCC 7469 type antigen was mainly associated with the physiological characters defining Briggs group IV: 22 of the organisms belonging to this serological type fell into her group IV, only 3 into group VI, and the remaining 4 into sections 3 and 4 . The strains having other type antigens, however, belonged mostly to group VI: 10 fell into this group and only 3 into group IV, whilst 3 were in sections 3 and 4. Although some overlapping occurs, the differentiation is clear enough to suggest that the Lactobacillus casei-helveticus group might be further divided to identify strains belonging to the serological type ATCC 7469 as $L$. helveticus, and strains belonging to other serological types as $L$. casei-helveticus. The ability of strains 
of $L$. helveticus to grow at high temperatures has important practical applications and a serological differentiation correlated with such a characteristic would be greatly valued. The examination of further strains is necessary, however, before the wider validity of such a distinction could be established.

Strains in the Lactobacillus casei-helveticus serological group which did not possess the ATCC 7469 type antigen consisted of 6 named strains of $L$. casei, 2 of $L$. helveticus, 2 of $L$. acidophilus, 1 of $L$. odontolyticus, 2 oral strains and $\mathbf{3}$ other strains isolated from milk and cheese. Strains belonging to serological type ATCC 7469 consisted of 10 named strains of $L$. helveticus, 10 of L. casei, 2 of $L$. acidophilus, 1 of $L$. bulgaricus, 1 of $L$. odontolyticus, 2 of $L$. delbrueckii, 1 oral strain and 2 freshly isolated strains from yoghourt and Stilton cheese respectively. Nine of the organisms in this latter section ( 3 of L. casei and 6 of $L$. helveticus), although received from various sources, are believed (Briggs, $1953 a$ ) to be cultures of $L$. casei ATCC 7469 as all bore this collection number. It is of interest to note that they have all retained their type and group antigens under diverse conditions of maintenance.

Table 3. Reciprocal absorption tests showing the antigenic relationship between strains of $\mathrm{L}$. casei and $\mathrm{L}$. helveticus

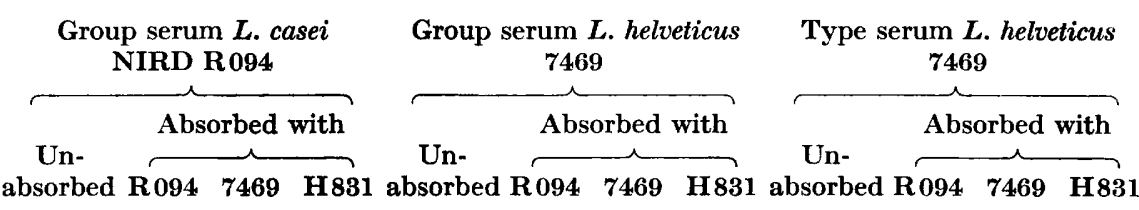

Strains tested $\begin{array}{lllllllll}\text { absorbed R } 094 & 7469 & \text { H831 absorbed R094 } & 7469 & \text { H831 absorbed R094 } & \mathbf{7 4 6 9} & \text { H831 }\end{array}$

L. casei

NIRD R094 15 other strains

L. helveticus ATCC 7469 28 other strains

L. casei

H831

$\begin{array}{llllllllllll}+ & - & + & + & + & - & - & + & - & - & - & - \\ + & - & - & + & + & - & - & + & - & - & - & - \\ + & - & - & + & + & + & - & + & + & + & - & + \\ + & - & - & + & + & + & - & + & + & + & - & + \\ & & & & & & & & & & & \end{array}$

\section{LACTOBACILLUS CASEI GROUP}

The Lactobacillus casei serological group is quite distinct from the L. caseihelveticus group; no cross-reactions occurred between sera of the two groups and absorption with cells of strain $\mathbf{H} 831$ did not remove the group antibodies from L. casei-helveticus sera (Table 3). Extracts of 14 strains of lactobacilli reacted with two group sera prepared against $L$. casei NIRD $\mathrm{H} 831$ and L. casei NIRD DECP respectively. Organisms included in this group consisted of 6 strains received as L. casei, 1 as L. lactis, 2 as L. plantarum (both having the same NCTC number but from different sources), 1 oral strain, and 4 freshly isolated strains from fresh and soured milk. Of these, 12 fell into Briggs group VI and 2 into her section 3.

\section{LACTOBACILLUS FERMENTI GROUP}

Twenty-two strains of lactobacilli reacted specifically with a serum prepared against a strain of Lactobacillus fermenti (L. fermentatus ATCC 9338; NCTC 
6991), and also with a serum prepared against a freshly isolated oral strain 010, which showed similar physiological characteristics. All the strains in this serological group were heterofermentative organisms, 21 being included in Briggs group VIII and 1 in Briggs group VII. However, 5 other strains identified by other workers as L. fermenti, and $\mathbf{3}$ freshly isolated strains also in Briggs group VIII, did not react with either of these sera. It was noted that 7 of these strains were of intestinal origin, whereas only 2 of the 22 reacting strains were intestinal. It is possible that there is another serological group consisting mainly of intestinal strains of $L$. fermenti as opposed to those of oral, milk and plant origin, although $I$ have not yet succeeded in preparing a corresponding serum. Strains in the L. fermenti group consisted of 10 strains named $L$. fermenti, 2 of $L$. gayonii, 1 of L. mannitopoeus, 1 of L. lycopersici, 4 freshly isolated strains from Gruyère cheese, 2 oral strains and 1 strain each from pig faeces and chick duodenum.

Prolonged inoculation of rabbits with organisms in this group led to the production of antibodies which reacted weakly with extracts of a number of strains of the Lactobacillus casei-helveticus group. Similarly, weak antibodies which reacted with extracts of $L$. fermenti could be detected in $L$. casei-helveticus antisera after long courses of inoculation, indicating a minor antigen common to these two groups.

\section{LACTOBACILLUS PLANTARUM GROUP}

Into this group fell 79 strains of lactobacilli, all of which reacted specifically with a serum prepared against Lactobacillus plantarum NCTC 7220. Attempts to prepare similar group sera against other laboratory or freshly isolated strains of $L$. plantarum, extracts of which reacted strongly with this serum, proved unsuccessful. All strains in this serological group fell into Briggs group VI, and included 17 strains named $L$. plantarum, 4 of $L$. arabinosus, 2 of $L$. pentosus, 3 of $L$. casei, 3 of $L$. odontolyticus, 2 of $L$. pastorianus and 1 of L. fermenti. Freshly isolated strains were obtained from silage, raw and soured milk, and Cheddar cheese.

It was noted that when inoculation of rabbits with strain NCTC 7220 continued for longer than was necessary to obtain a Lactobacillus plantarum group antiserum other antibodies were detected in the serum. Only extracts of strains of $L$. plantarum and of organisms of similar physiological characteristics reacted with early bleedings, after a further course of inoculation extracts of strains of organisms of the $L$. lactis-brevis group (described below) also reacted. It was possible to remove this cross-reaction by absorption with a strain of $\boldsymbol{L}$. lactis or $\boldsymbol{L}$. brevis but the specific reaction was then also somewhat weakened, and it was found advisable to prepare group sera by using as short a course of inoculations as possible. It appeared that the plantarum strain possessed a minor antigen in common with $L$. lactis and $L$. brevis; this was confirmed by further work on these two organisms.

\section{LACTOBACILLUS LACTIS-BREVIS GROUP}

Using the Lactobacillus lactis strain Dorner type I (ATCC 8000, NCTC 7278) a group serum was prepared for the L. lactis serological group. Another strain, 
L. lactis NIRD 244, stimulated similar but more potent antisera. Reciprocal absorption tests showed these two organisms to be identical. Eighty-three strains reacted specifically with these sera, including 18 of 19 strains received as $L$. lactis, 5 received as $L$. bulgaricus, 2 as $L$. helveticus and 1 as $L$. acidophilus. Freshly isolated strains were from calf abomasum, présure, dried calf stomach, raw milk, Gruyère cheese. Of these strains 70 fell into Briggs group $V$, 2 into group III, 8 into section 1 , and 1 into section 4 . Thus strains in this serological group were all homofermentative and mainly thermophilic organisms, unable to hydrolyse arginine, able to grow at $48^{\circ}$ and to resist heating to $60^{\circ}$ for $1 \frac{1}{2} \mathrm{hr}$. (Table 1). It seemed that another serological group containing only organisms closely allied in physiological characteristics had

Table 4. Reciprocal absorption tests showing antigenic relationship between strains belonging to the L. lactis and L. brevis serological group

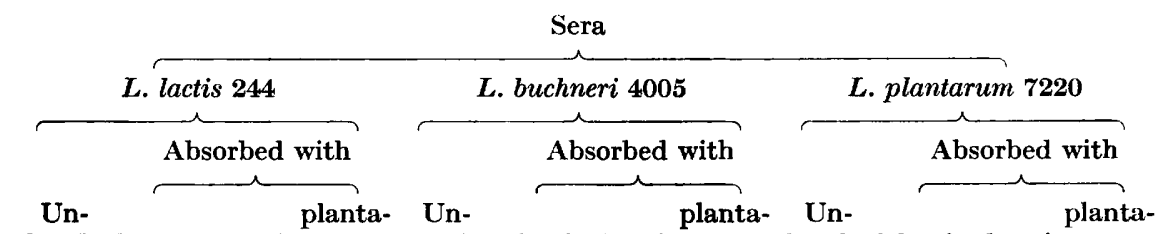

Strains tested absorbed lactis brevis rum absorbed lactis brevis rum absorbed lactis brevis rum

12 of $L$. lactis

8 of $L$. brevis

$++\quad-\quad+++$

5 of $\boldsymbol{L}$. plantarum

been established. However, it was observed that these otherwise specific sera gave strong cross-reactions with another group of organisms which differed markedly from the $L$. lactis group in physiological reactions, being heterofermentative, hydrolysing arginine, growing at $15^{\circ}$ but not at $45^{\circ}$, and unable to withstand heating to $60^{\circ}$ for $30 \mathrm{~min}$. In contrast with the rapid growth of the $\boldsymbol{L}$. lactis organisms they grew very slowly. All these heterofermentative strains fell into Briggs group VII, and included named strains of $L$. brevis, L. acidophil-aerogenes, L. bifidus, L. rudensis, L. buchneri, L. hilgardii, L. caucasicus, L. kefir, L. frigidus, L. malefermentans, L. parvus and 13 strains of L. pastorianus. Freshly isolated strains were obtained from beer, silage, raw and soured milk, yoghourt and Cheddar cheese. It would scarcely be possible to find within the lactobacillus genus two groups more divergent biochemically, and yet they appeared to possess a common major antigen. It was not, as with the $L$. plantarum group, the question of a shared minor antigen giving rise to slight or delayed cross-reacting antibodies in the sera, but extracts of these heterofermentative strains in the $L$. brevis group reacted as strongly as extracts of the $L$. lactis group with the $L$. lactis sera, and at the same stage of preparation of the antisera. Sera were prepared against two of the heterofermentative organisms, L. brevis ATCC 367 (NCIB 8169) and L. buchneri ATCC 4005 (NCIB 8007). Both these sera also gave strong reactions with extracts of organisms of the $L$. lactis serological group, as well as with those of the $L$. brevis group. The reciprocal absorption tests (Table 4 ) between $L$. lactis 
NIRD 244 and L. buchneri ATCC 4005, and their antisera showed complete cross-absorption. Various organisms were used in an endeavour to obtain differential absorption between sera against the two groups of organisms, and partial success was eventually obtained by absorbing with $L$. plantarum NCTC 7220. It was found that this strain absorbed antibodies which reacted with the extracts of the $L$. brevis strains, whereas the $L$. lactis extracts still reacted, although not so strongly. Table 4 shows that sera prepared against either $L$. lactis or $L$. brevis, after absorption with $L$. plantarum NCTC 7220 cells, reacted only with the $L$. lactis strains and not with the $L$. brevis strains. This absorption was not easy to accomplish: when the proportion of cells to serum was too small, extracts of the L. brevis strains also reacted with the serum; when too great, the precipitin reaction for the $L$. lactis strains was greatly weakened. Other strains of $L$. plantarum had a similar effect, but strain NCTC 7220 was found to give the best results. By means of this quantitative absorption it was possible to separate these two groups.

Table 5 gives a summary of the distribution of the physiological groups of Briggs among the serological groups of lactobacilli found in the present work.

\section{UNCLASSIFIED STRAINS}

Of the strains examined here $70 \%$ have been classified into serological groups. The remaining 130 organisms have not yet been identified serologically. Of these, 73 named strains included 17 strains of Lactobacillus acidophilus, 8 of $L$. delbrueckii, 5 of $L$. bulgaricus, 6 of L. leichmannii, 5 of L. fermenti, 5 of L. helveticus, 2 of L. jugurt, 7 of $L$. casei, 11 of L. plantarum, 2 of L. pentoaceticus, and 1 each of L. lactis, L. arabinosus, L. brevis, L. bifidus and L. lycopersici. Fifty-seven freshly isolated strains also remained unclassified, 34 from intestinal sources, and the remaining 23 from silage, milk, cheese, greening sausage, and sauerkraut. The distribution of these serologically unclassified strains among the Briggs physiological groups is shown in Table 5.

Table 5. Association of physiological characteristics with the serological grouping in 442 strains of lactobacilli

Number of strains falling into each of the Briggs physiological groups

\begin{tabular}{|c|c|c|c|c|c|c|c|c|c|c|c|c|c|c|}
\hline \multirow{3}{*}{$\begin{array}{c}\text { Serological } \\
\text { group }\end{array}$} & & & & & & & & & & & & & & \\
\hline & & \multicolumn{6}{|c|}{ Physiological group } & & \multicolumn{4}{|c|}{ Section } & & \\
\hline & I & II & III & IV & $\mathbf{V}$ & VI & VII & VIII & 1 & 2 & 3 & 4 & $\begin{array}{l}\text { Not } \\
\text { tested }\end{array}$ & Total \\
\hline bulgaricus & 5 & 8 & 9 & . & 2 & 1 & . & . & . & . & . & 1 & . & 26 \\
\hline casei-helveticus & . & . & . & 25 & . & 13 & . & . & . & . & 5 & 2 & . & 45 \\
\hline casei & . & . & . & . & . & 12 & . & . & . & . & 2 & . & . & 14 \\
\hline plantarum & . & . & . & . & . & 79 & . & . & . & . & . & . & . & 79 \\
\hline lactis & . & . & 2 & . & 70 & . & . & . & 8 & . & . & 1 & 2 & 83 \\
\hline brevis & . & . & . & . & . & 42 & . & . & . & . & . & . & 1 & 43 \\
\hline fermenti & . & . & . & . & . & . & 1 & 21 & . & . & . & . & . & 22 \\
\hline Not grouped & 28 & 8 & 9 & . & 8 & 22 & 12 & 8 & 12 & 9 & 4 & 6 & 4 & 130 \\
\hline
\end{tabular}

Reproducibility of results

Extracts of all strains of lactobacilli were again made at least a year after first testing, and retested against the same sera and against fresh sera prepared 
with the same strains. With a few exceptions precipitin tests gave the same serological reactions as on first testing. Nine strains of the Lactobacillus plantarum serological group (including 7 freshly isolated ones), which had originally reacted strongly with the serum, reacted only weakly or not at all on retesting, and one strain of $L$. casei-helveticus group was found to have lost both type and group antigens. None of these strains had acquired the antigens of any other group. Freshly isolated strains tested on first isolation and again after laboratory cultivation were, with the exception of the strains quoted above, unchanged serologically as far as the group antigen was concerned.

It has recently been found that when one unidentified strain was used to prepare a group serum, this reacted with acid extracts of all strains within the Lactobacillus casei group, but not with the homologous extract. It was then found that by treating the cells of the homologous organism by other methods, such as autoclaving at $120^{\circ}$ for 5 min. (Rosendal, 1950), grinding, or use of the tissue disintegrator, it reacted strongly with the homologous antiserum. The reason for this occasional failure of $\mathrm{HCl}$ extraction, which did not affect other strains of the same serological type, is not yet known.

\section{Crossing antigen $\boldsymbol{R}$}

Mention has already been made of minor antigenic cross-reactions which occurred between organisms in different serological groups. In addition to these a major antigen (named $\mathrm{R}$ by the author) was noted in a number of organisms of different serological groups. Sera containing $\mathbf{R}$ antibodies reacted selectively with a number of strains belonging to different groups, giving a strong precipitin reaction. Although its distribution appeared to be random, extracts of all strains were tested for its presence, as further work may indicate some biochemical characteristic common to all these strains. Sera against this antigen were prepared by using a strain of an unidentified Lactobacillus sp., AH 4, isolated from Gruyère cheese and falling into section 1 of Briggs, and a strain of L. bulgaricus SM 10 isolated from soured milk. The 37 strains in which this antigen was demonstrated fell into the following serological groups: 8 in L. bulgaricus, 2 in L. casei, 9 in L. lactis, 3 in L. fermenti and 2 in $L$. plantarum group; the other 13 strains were unidentified serologically. Most of the identified strains were members of the L. bulgaricus and L. lactis serological groups, but strains from all other groups except $L$. caseihelveticus and $L$. brevis were represented. It is interesting that although the origin of some of these strains is not known, 20 of them were known to be isolated from milk or milk products, and only one was of intestinal origin.

\section{Ethanol precipitation of crude extracts}

On testing fractions A (ethanol insoluble) and B (ethanol soluble), prepared by Harrison's method (Harrison et al. 1939; see Methods) against group and type sera it was found that the group antigen was precipitated by ethanol; only fraction A reacted with the group sera. This agrees with similar findings by Shattock (1949) with group D streptoccoci that the group antigen was contained in the ethanol precipitated fraction. The type substance was present in 
both fractions. This method of concentrating the group antigen was tried with several ungrouped strains, but in no case did it reveal the presence of group antigen not already indicated in the crude unconcentrated extracts.

\section{Comparison of precipitin and agglutination tests}

Early in this work the results of agglutination tests were compared with those of precipitin tests, and it was found that agglutination tests indicated much narrower relationships among the lactobacilli than the precipitin reactions, and probably indicated type antigens. Later, a few strains of each group were tested against their group sera by agglutination test and the results confirmed the findings of the earlier work. Strains belonging to the same serological group but having different type antigens reacted only weakly in agglutination tests, usually to a titre of less than $1 / 80$, to potent precipitating group sera. It appears that, as with the streptococci (Lancefield, 1941), the group reactions are not indicated by agglutination reactions. It was also found that the type reactions were not entirely in agreement in the two tests. Even when homologous type sera were used, the presence of a high agglutinin titre in a serum was not always associated with a strong precipitin reaction, and several type sera which gave rapid and massive precipitin reactions were only of low agglutinin titre. This suggests the presence of more than one type antigen, the presence of which is indicated only by the appropriate test, analogous with the $\mathbf{M}$ and $\mathbf{T}$ antigens of the group $\mathbf{A}$ streptococci.

\section{Comparison with results of other workers}

Few comparisons could be made with the serological results of other workers, since so little extensive serological work has been done with lactobacilli. However, Dr Orland kindly supplied me with 7 of the strains identified by Orland (1950) or by Williams (1948a) as possessing some of the antigenic components they mentioned. Table 6 shows a comparison of my serological grouping with their findings; 4 of Orland's strains fall into the Lactobacillus casei-helveticus group ( 2 of them being L. helveticus), 2 are L. plantarum and 1 is L. casei. As might be expected, there is little agreement between my precipitin and Orland's agglutination tests, which do not indicate the group relations between his organisms. It is of interest that the two $L$. helveticus strains (of which $L 320$ bears the number ATCC 7469) were the strains with the F antigen, and it seems very probable that this $\mathbf{F}$ antigen may correspond to the $L$. helveticus type antigen. Orland mentioned that of the 30 strains which possessed this antigen, 9 were labelled ATCC 7469 and were presumably cultures of the same organism from different laboratories. In this work it has already been mentioned that 9 of the strains received here as either $L$. casei or $L$. helveticus were believed to be strains of this organism, and all of these were identified as L. helveticus. The other antigens of Orland and of Williams shown by agglutination tests are presumably type antigens, and are not indicated in the precipitin tests, i.e. $45 \mathrm{~A}$ is not related to $3 \mathrm{~A} 1 \mathrm{SB}$ although both possess Orland's $B$ antigen. 
Table 6. A comparison of the serological classifications of certain lactobacilli by Orland and by Williams and in the present work

\begin{tabular}{|c|c|c|c|}
\hline \multirow[b]{2}{*}{ Strain } & \multirow[b]{2}{*}{$\begin{array}{c}\text { Source of } \\
\text { identification }\end{array}$} & \multicolumn{2}{|c|}{ Serological classification } \\
\hline & & $\begin{array}{c}\text { Orland and Williams } \\
\text { antigen }\end{array}$ & $\begin{array}{l}\text { Serological group of } \\
\text { present work }\end{array}$ \\
\hline $3 \mathrm{~A} 1 \mathrm{SB}$ & Oral & $\mathrm{AB}$ & casei-helveticus \\
\hline $45 \mathrm{~A}$ & Oral & $\mathrm{BD}$ & casei \\
\hline EH22 G & Oral & $\mathbf{E}$ & plantarum \\
\hline L320 ATCC 7469 & L. casei & $\mathbf{F}$ & casei-helveticus $\mathrm{H}^{*}$ \\
\hline L304 & L. plantarum & $\mathbf{G}$ & plantarum \\
\hline L 323 & L. casei & $\mathbf{H}$ & casei-helveticus \\
\hline L641 & Oral & Fl & casei-helveticus $\mathbf{H}^{*}$ \\
\hline
\end{tabular}

\section{Serological identity of differing colonial forms}

It is of interest that my colleague Dr D. M. Wheater plated out all the $\mathbf{4 4 2}$ strains examined here and found at least two widely different colonial forms in a number of strains. When the different variants of 25 strains were examined serologically, it was found in each case that the group antigen was the same for all the colonial variations in a strain; 1 strain exhibited four different colony forms, but extracts of each reacted with the same group serum.

\section{DISCUSSION}

Most of the difficulties mentioned in the preparation of the lactobacillus grouping sera are generally known to occur in the preparation of immune sera, particularly with the streptococci: Briggs \& Newland (1952) mentioned the presence of antibodies which reacted with extracts of groups $\mathrm{L}$ and $\mathrm{K}$ streptococci in group $\mathrm{N}$ sera prepared against Streptococcus cremoris. With the lactobacilli the ease with which such minor cross-reacting antibodies develop, and the greater difficulty in preparing group sera with freshly isolated strains may have prevented this method of classification being used in the past. Once such specific group sera have been prepared the rapid identification of large numbers of lactobacilli presents no difficulty, the preparation of the crude extracts being quick and simple, and the troubles of the agglutination test, caused by unstable suspensions and autoagglutination, are avoided. The fact that the group antigens were present in old laboratory strains, which were as readily grouped as freshly isolated strains, showed them to be stable antigens and therefore suitable for serological identification. In this connexion it was noted that when several strains bearing the same code number or name were obtained from different laboratories, all such replicate cultures gave identical serological reactions; different methods of maintenance had not affected their specific reactions.

A similar division into broad groups forms the basis of successful serological classification of another member of the Lactobacteriaceae, the streptococci (Lancefield, 1933), and in view of the many characteristics shared by the two genera, it is not surprising that their serological attributes should be similar. 
It is clear that lactobacilli possess a complexity of antigens, as Canby \& Bernier (1942) concluded. In addition to the type and group antigens certain strains also have the $\mathbf{R}$ antigen, minor cross-reactions occur between groups, and there is evidence that other cross-reactions may occur selectively and yet unpredictably, cutting across the groups. It has been the practice to avoid preparing group sera from strains which contain such antigens, and to concentrate on the classification into broad groups.

The close agreement attained between the serological grouping and the physiological tests of Briggs is indicated in Table 5. That the two methods of classification were carried out concurrently and independently emphasizes the agreement between the serological and physiological findings. Each serological group is almost entirely represented in one physiological group, except that the Lactobacillus bulgaricus serological group organisms are distributed over the Briggs physiological groups I, II and III, and that strains in the L. casei-helveticus serological group occur in physiological groups IV and VI. It has also been possible to divide the large physiological group VI into three serological groups, L. casei-helveticus, L. casei and $L$. plantarum. Briggs (1953a) herself considered that physiological groups I, II and III, though plainly separated from the remaining groups, were not so clearly distinct from each other and that further confirmatory tests were necessary to distinguish them; Wheater (1955), in her work on the physiological characteristics of L. acidophilus and $L$. bulgaricus, has shown that the distribution of strains within the Briggs groups I-III is not the same as the distribution within the two species, so that strains of all three groups occurred in both L. acidophilus and $L$. bulgaricus. A close agreement was found between Wheater's defined species $L$. bulgaricus and the present serological group $L$. bulgaricus. In the case of $L$. casei-helveticus serological group, with strains distributed into two of the Briggs groups, it has been shown that it is possible to subdivide this serological group into $L$. casei-helveticus (containing mostly group VI strains) and $L$. helveticus (containing mostly group IV strains).

Thirty per cent of the strains tested still remain unclassified serologically. It will be noted that no mention has been made of a serological group corresponding to Lactobacillus acidophilus, and in fact 17 of the 22 strains received as L. acidophilus, and 23 homofermentative freshly isolated strains of intestinal origin, also probably $L$. acidophilus, remain as yet unidentified serologically. It has not yet been possible to prepare an antiserum which reacts with these strains. In some instances sera have been obtained which reacted specifically with some extracts, but in each case the number reacting was small, and may have been due to type reactions. Two such groups were defined among the intestinal lactobacilli, each containing 6 strains, but it was considered that neither group was large enough to constitute a named serological group. It may be that intestinal lactobacilli present peculiar problems in their serological grouping; it has been mentioned that several intestinal strains of $L$. fermenti could not be classified. The grouping of these organisms needs further work to determine their position.

None of the 6 strains of Lactobacillus leichmannii has been grouped, attempts 
to produce a serum against one of them having been unsuccessful, and no group for $L$. delbrueckii has been defined. These may constitute further serological groups. Some unidentified strains may also have lost their group antigen; such loss of antigenicity has been experienced, particularly with strains of $\boldsymbol{L}$. plantarum, although group antigen is normally very stable.

The close serological relationship of the biochemically divergent groups Lactobacillus lactis and $L$. brevis is of interest. Davis (1936) suggested that ecologically there were two broad groups of lactic acid bacteria, plant strains and animal strains, and that the use by man of milk, fermented mashes, and alcoholic drinks were artificial procedures which might select new species originating, however, from one of the two broad groups. Such a theory might account for a common origin of $L$. brevis and $L$. lactis, the two groups later becoming adapted to very different conditions. On the other hand, the sharing of antigenic components among different species or even genera of bacteria is not uncommon, e.g. the polysaccharide shared by pneumococcus type II and Leuconostoc mesenteroides (Sugg \& Hehre, 1942), the polysaccharide common to pneumococcus type VI and Haemophilus influenzae type A (Chapman \& Osborne, 1942), and a common type antigen shared between group D and group N streptococci (Sharpe, 1952). It seems however that, in view of this close relationship between homofermentative and heterofermentative groups of lactobacilli, it would be unwise to separate the homofermentative from the heterofermentative species, placing the latter in a separate genus as suggested by Rogosa, Wiseman, Mitchell, Disraely \& Beaman (1953).

Harrison's (Harrison et al. 1939; Harrison \& Opal, 1944) work on oral and intestinal strains of lactobacilli, with the use of purified $\mathrm{HCl}$ extracts, apparently indicated type and not group antigens. This was probably due to several causes: (i) as he used quantitative precipitin tests the group antigen, being much weaker than the type, would be diluted out more readily and the group reaction, when present, would only occur in the higher concentrations of antigen and be considered of minor importance; (ii) cultures used for preparing sera had been isolated within the previous 12 months and would have tended to stimulate the production of type rather than group antibodies; (iii) the inoculating suspensions were heated to $65^{\circ}$ for $1 \mathrm{hr}$., and this has been found, at least in some cases, partially to destroy the group antigen.

The definition of these serological groups, substantiated by physiological tests and compatible with sources of origin, should remove much of the confusion existing in the serological relationships of the lactobacilli and serve as a basis for further elucidating their complex antigenic patterns.

I wish to thank Dr A. T. R. Mattick for his helpful advice and many valuable suggestions; also Mr R. W. Goswell and Miss P. Burrows for technical assistance. 


\section{REFERENCES}

Briggs, C. A. E. \& Newland, L. G. M. (1952). The serological classification of Streptococcus cremoris. J. Dairy Res. 19, 160.

BrigGs, M. (1953a). The classification of lactobacilli by means of physiological tests. J. gen. Microbiol. 9, 234.

Briggs, M. (1953b). An improved medium for lactobacilli. J. Dairy Res. $20,36$.

Canby, C. P. \& Bernier, J. L. (1942). Bacteriologic and immunologic studies on dental caries: a preliminary report. J. Amer. dent. Ass. 29, 606.

Chapman, O. D. \& Osborne, W. (1942). Serological relationships between Diplococcus pneumoniae and Hemophilus influenzae. J. Bact. 44, 620.

Davis, J. G. (1936). A procedure for the isolation and identification of the lactic acid bacteria. Proc. Soc. agric. Bact. 1936.

VAN DEN ENDE, M. (1952). Observations on the antigenic structure of Pseudomonas aeruginosa. J. Hyg., Camb. 50, 405.

Fuller, A. T. (1938). The formamide method for the extraction of polysaccharides from haemolytic streptococci. Brit. J. exp. Path. 19, 130.

Harrison, R. W. \& Opal, Z. Z. (1944). Comparative studies on lactobacilli isolated from the mouth and intestine. J. dent. Res. $23,1$.

Harrison, R. W., Zidek, Z. C. \& Hemmens, E. S. (1939). Studies on lactobacilli II. Type specific immunological reactions of oral strains. J. infect. Dis. 65, 255.

$J_{\mathrm{ACOB}}, \mathbf{M}$. M. (1947). Investigations on streptococcal mastitis and the streptococci. University Reading: Thesis.

LANCEFIELD, R. C. (1933). A serological differentiation of human and other groups of hemolytic streptococci. J. exp. Med. 57, 571.

LaNCEFIELd, R. C. (1941). Specific relationship of cell composition to biological activity of hemolytic streptococci. Harvey Lect. 36, 251.

Mickle, H. (1948). Tissue disintegration. J. R. micr. Soc. 68, 10.

Niven Jun., C. F., Castellani, A. G. \& Allanson, V. (1949). A study of the lactic acid bacteria that cause surface discolourations of sausages. J. Bact. 58, 633 .

OrLAND, F. J. (1950). A correlation of antigenic characteristics among certain bacteria of the lactobacillus group. J. infect. Dis. 86, 63 .

Rogosa, M., Wiseman, R. F., Mitchell, J. A., Disraely, M. N. \& Beaman, A. J. (1953). Species differentiation of oral lactobacilli from man including descriptions of Lactobacillus salivarius nov. spec. and Lactobacillus Cellobiosus nov. spec. J. Bact. 65, 681.

Rosendal, K. (1950). A new method for preparing type specific extracts from haemolytic streptococi of group A, Type 2. Nature, Lond. 166, 912.

Sharpe, M. E. (1952). Occurrence of a common type antigen in streptococci of groups D and N. J. gen. Microbiol. 7, 192.

Shatтock, P. M. F. (1949). The streptococci of group D; the serological grouping of Streptococcus bovis and observations on serologically refractory group D strains. J. gen. Microbiol. 3, 80.

Sugg, J. Y. \& Hehre, E. J. (1942). Reactions of Leuconostoc mesenteroides with the antiserums of Leuconostoc and of types 2, 20 and 12 Pneumococcus. J. Immunol. 43, 119 .

Wheater, D. M. (1955). The characteristics of Lactobacillus acidophilus and Lactobacillus bulgaricus. J. gen. Microbiol. 12, 123.

Williams, N. B. (1948a). Antigenic components of lactobacilli of human oral origin. J. infect. Dis. 82, 31.

Williams, N. B. (1948b). Immunologic reactions of lactobacilli. J. Amer. dent. Ass. $37,403$.

(Received 24 August 1954) 